\section{Arrkivoc

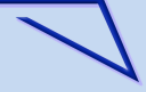

Archive for

Organic Chemistry
The Free Internet Journal

for Organic Chemistry
Paper

Arkivoc 2018, part v, 0-0

\title{
Green synthesis of highly functionalized octahydropyrrolo[3,4-c]pyrrole derivatives using subcritical water, and their anti(myco)bacterial and antifungal activity
}

\author{
Yahya Nural, ${ }^{* a}$ Muge Gemili, ${ }^{a}$ Erdal Yabalak, ${ }^{b}$ Laurens M. De Coen, ${ }^{\mathrm{c}}$ and Mahmut Ulger ${ }^{\mathrm{d}}$
}

${ }^{a}$ Department of Analytical Chemistry, Faculty of Pharmacy, Mersin University, Mersin, TR-33169 Turkey

${ }^{b}$ Department of Chemistry, Faculty of Arts and Science, Mersin University, Mersin, TR-33343 Turkey

'Department of Green Chemistry and Technology, Faculty of Bioscience Engineering, Ghent University, Ghent, B-9000 Belgium

${ }^{d}$ Department of Pharmaceutical Microbiology, Faculty of Pharmacy, Mersin University, Mersin, TR-33169

Turkey

Email:ynural1805@yahoo.com

Received 03-16-2018

Accepted 04-30-2018

Published on line 06-03-2018

\section{Abstract}

A series of novel 2-(thiazol-2-yl)-octahydropyrrolo[3,4-c]pyrroles was synthesized by reaction of octahydropyrrolo[3,4-c]pyrrole $\mathrm{N}$-benzoylthiourea derivatives and $\alpha$-haloketones in subcritical water at $130{ }^{\circ} \mathrm{C}$ in $75-91 \%$ yield. Both the thiourea intermediates and the end products were synthesized in subcritical water, which proved a suitable green alternative to acetone by delivering the desired compounds in much shorter reaction times and practically the same yields. The antimicrobial activity of the compounds was determined against five bacterial strains and three fungal strains, and MIC values of $15.62-250 \mu \mathrm{g} / \mathrm{mL}$ were observed. Moreover, the compounds exhibited antimycobacterial activity against $M$. tuberculosis $H 37 R v$ with MIC values of $7.81-62.5 \mu \mathrm{g} / \mathrm{mL}$.<smiles>[R]N1C(=O)C2C(C(=O)OC)NC(c3ccccc3)(c3ccccc3)[C@H]21</smiles>

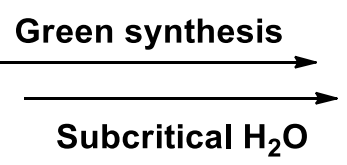<smiles>[R]C(=O)c1cnc(N2C(C(=O)OC)C3C(=O)N([R])C(=O)C3C2(c2ccccc2)c2ccccc2)s1</smiles>

Keywords: Green chemistry, subcritical water, thiazole, pyrrolidine, pyrrolidinedione, antimicrobial 


\section{Introduction}

Thiazole derivatives containing nitrogen and sulphur atoms are among the most important pharmacophore groups in drug research studies ${ }^{1}$ and it is known that many drugs ${ }^{1-3}$ and natural products ${ }^{4}$ such as Abafungin, Famotidin, Meloxicam and Ritonavir contain a thiazole ring in their structures. ${ }^{1-4}$ Thiazoles are present in a wide range of pharmacologically active compounds exhibiting antibacterial, ${ }^{5-8}$ antimycobacterial, ${ }^{9-11}$ antifungal, ${ }^{5-8}$ anticancer ${ }^{12-14}$ and antiviral ${ }^{15,16}$ activity. Furthermore, thiazole derivatives are useful as chemosensors ${ }^{17}$ and fluorescent sensors. ${ }^{18}$

The pyrrolidine moiety has been intensively studied in medicinal chemistry ${ }^{19}$ due to its presence in the structure of many biologically active compounds such as anisomycin ${ }^{20}$ and hygrine. ${ }^{21}$ Furthermore, pyrrolidine derivatives show various pharmacological activities such as antimicrobial, ${ }^{22-24}$ antiviral ${ }^{25}$ and anticancer activity. ${ }^{26,27}$ In addition, pyrrolidine-2,5-dione derivatives have also been reported to exhibit a wide range of pharmacological activities such as antimicrobial ${ }^{28}$ and anticonvulsant activity. ${ }^{29}$ Compounds containing pyrrolidine fused to pyrrolidine-2,5-dione represent an important class of bicyclic ring systems in drug research and exhibit various pharmacological activities such as antibacterial ${ }^{30,31}$ and anticancer activity. ${ }^{32}$

Subcritical water is a green alternative to conventional solvents due to being an environmentally friendly, cheap, safe and non-toxic solvent. ${ }^{33}$ Water, which is heated at a temperature range of $100{ }^{\circ} \mathrm{C}-374.2^{\circ} \mathrm{C}$ and pressurized enough to keep it in the liquid state in this range is defined as subcritical water. ${ }^{34}$ Subcritical water offers the unique medium for various processes such as oxidation, extraction, ${ }^{35,36}$ solubility ${ }^{37}$ and organic

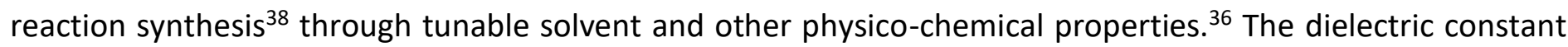
that determines the polarity of a solvent can be easily reduced by increasing the temperature in subcritical water. ${ }^{39}$ Thus, subcritical water acts as a non-polar solvent like ethanol or methanol, providing a favorable medium for organic synthesis. Many reactions such as alkylation, condensation, coupling, decomposition, DielsAlder, decarboxylation, dehydration, elimination reactions and nano-synthesis can be carried out using subcritical water. ${ }^{38,40,41}$

In this study, we designed and synthesized octahydropyrrolo[3,4-c]pyrrole $N$-benzoylthioureas and a series of novel 2-(thiazol-2-yl)-octahydropyrrolo[3,4-c]pyrrole derivatives in subcritical water as green solvent, and investigated their anti(myco)bacterial and antifungal activities.

\section{Result and Discussion}

The synthesis of octahydropyrrolo[3,4-c]pyrrole derivatives $\mathbf{2} \mathbf{a}-\mathbf{b}$ was performed according to a literature method $^{42}$ via a 1,3-dipolar cycloaddition reaction of methyl 2-(diphenylmethyleneamino)acetate and $\mathrm{N}$ methylmaleimide or $N$-phenylmaleimide in $85 \%$ and $82 \%$ yield, respectively.

The synthesis of the octahydropyrrolo[3,4-c]pyrrole $N$-benzoylthiourea derivatives 3a-b and 2-(thiazol-2-yl)octahydropyrrolo[3,4-c]pyrrole derivatives $4 a-j$ in subcritical water was performed in a reaction apparatus as depicted in Figure 1. The experiments were carried out in a home-made stainless steel reactor with an internal volume of $200 \mathrm{~mL}$ equipped with a heater-magnetic stirrer. To synthesize the octahydropyrrolo[3,4-c]pyrrole $\mathrm{N}$ benzoylthiourea derivatives 3a-b, the reactor was loaded with octahydropyrrolo[3,4-c]pyrroles $2 \mathbf{a}-\mathbf{b}$ (1 mmol), benzoyl isothiocyanate $(2 \mathrm{mmol}$ ) and $75 \mathrm{~mL}$ of ultra-pure water. After purging the reactor air with nitrogen, the internal pressure of the reactor was fixed at 30 bar supplied by nitrogen. Each experiment was performed at $130{ }^{\circ} \mathrm{C}$ for 4 hours under stirring. Finally, the reactor was depressurized and the obtained mixture was extracted 
using dichloromethane. The crude product was purified by column chromatography (EtOAc:hexane / 1:3) to afford compounds $3 \mathbf{3}-\mathbf{b}$ in $80-82 \%$ yield (Scheme 1, Table 1 ).

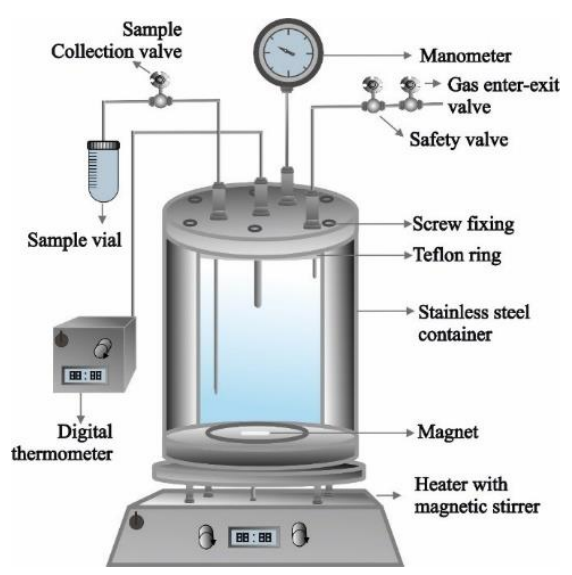

Figure 1. Schematic representation of the reactor equipped with a heater-magnetic stirrer.

For the synthesis of 2-(thiazol-2-yl)-octahydropyrrolo[3,4-c]pyrrole derivatives $4 a-j$ in subcritical water, a similar procedure described above was applied. Briefly, the reactor was loaded with octahydropyrrolo[3,4c]pyrrole $N$-benzoylthiourea 3 a or $\mathbf{3 b}(1 \mathrm{mmol})$, an appropriate $\alpha$-haloketone $(1.2 \mathrm{mmol})$ and $75 \mathrm{~mL}$ of ultrapure water. After purging the air in the reactor with nitrogen, the internal pressure of the reactor was fixed at 30 bar supplied by nitrogen. Each experiment was performed at $130{ }^{\circ} \mathrm{C}$ for 2 hours under stirring. Finally, the reactor was depressurized and the obtained mixture was extracted using dichloromethane. The crude product was purified by column chromatography (EtOAc:hexane / 1:4) to afford compounds 4a-j in 74-91\% yield (Scheme 1, Table 1).

Furthermore, the synthesis of the octahydropyrrolo[3,4-c]pyrrole $N$-benzoylthiourea derivatives $3 a-b$ and the 2-(thiazol-2-yl)-octahydropyrrolo[3,4-c]pyrrole derivatives 4a-j in subcritical water was compared with their synthesis in conventional organic solvent. The synthesis of compounds $\mathbf{3} \mathbf{a}-\mathbf{b}$ was performed in acetone at reflux temperature for 30 hours and the products were obtained in $80-82 \%$ yield. Similarly, the synthesis of compounds 4a-j was also performed in acetone at reflux temperature for 18 hours and the products were obtained in 67$89 \%$ yield. Thus, the synthesis in subcritical water has practically the same yields but has the advantages of a shorter reaction time and of being a green solvent. It can be said that subcritical water is an excellent alternative to conventional organic solvent for the synthesis of both octahydropyrrolo[3,4-c]pyrrole $N$-benzoylthiourea and 2-(thiazol-2-yl)-octahydropyrrolo[3,4-c]pyrroles.

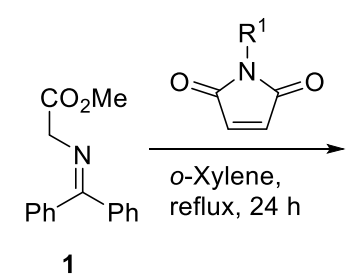

1

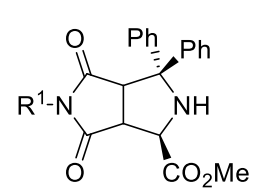

2a. $\mathrm{R}^{1}=\mathrm{Me}, 85 \%$ 2b. $R^{1}=P h, 82 \%$

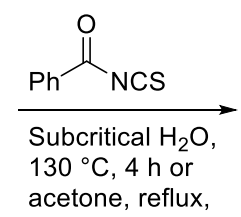

$30 \mathrm{~h}$

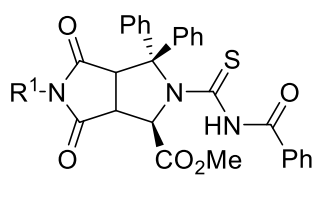

3a-b

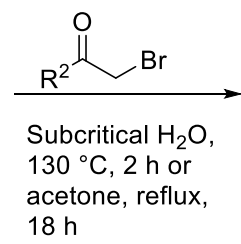

$18 \mathrm{~h}$

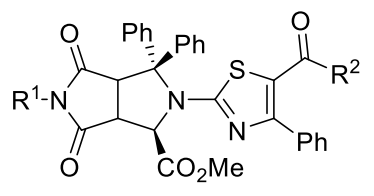

4a-j

Scheme 1. Synthesis of the 2-(thiazol-2-yl)-octahydropyrrolo[3,4-c]pyrrole derivatives. 
Table 1. Structure and yield of compounds 3 and $\mathbf{4}$

\begin{tabular}{|c|c|c|c|c|}
\hline Entry & $\mathrm{R}^{1}$ & $R^{2}$ & \multicolumn{2}{|c|}{ Yield $(\%)^{a}$ Yield $(\%)^{b}$} \\
\hline $3 a$ & Me & - & 86 & 82 \\
\hline $3 b$ & $\mathrm{Ph}$ & - & 80 & 80 \\
\hline $4 a$ & Me & $\mathrm{Ph}$ & 78 & 67 \\
\hline $4 b$ & Me & $4-\mathrm{CN}-\mathrm{C}_{6} \mathrm{H}_{4}$ & 90 & 84 \\
\hline $4 c$ & Me & $4-\mathrm{Br}-\mathrm{C}_{6} \mathrm{H}_{4}$ & 83 & 68 \\
\hline $4 d$ & Me & $2,4-\mathrm{Cl}_{2}-\mathrm{C}_{6} \mathrm{H}_{4}$ & 84 & 75 \\
\hline $4 e$ & Me & & 91 & 85 \\
\hline $4 f$ & $\mathrm{Ph}$ & $\mathrm{Ph}$ & 74 & 76 \\
\hline $4 g$ & $\mathrm{Ph}$ & $4-\mathrm{CN}-\mathrm{C}_{6} \mathrm{H}_{4}$ & 88 & 80 \\
\hline $4 h$ & $\mathrm{Ph}$ & 4-Br- $\mathrm{C}_{6} \mathrm{H}_{4}$ & 75 & 71 \\
\hline $4 \mathbf{i}$ & $\mathrm{Ph}$ & $2,4-\mathrm{Cl}_{2}-\mathrm{C}_{6} \mathrm{H}_{4}$ & 81 & 76 \\
\hline $4 j$ & $\mathrm{Ph}$ & & 90 & 89 \\
\hline
\end{tabular}

${ }^{a}$ Yield of the reaction in subcritical water. ${ }^{b}$ Yield of the reaction in acetone.

The antimicrobial activity screening of the octahydropyrrolo[3,4-c]pyrrole $\mathrm{N}$-benzoylthiourea derivatives 3ab and 2-(thiazol-2-yl)-octahydropyrrolo[3,4-c]pyrrole derivatives 4a-j was performed against five standard bacterial strains, one standard mycobacterium strain and three standard fungal strains. The compounds $3 a-b$ and $\mathbf{4 a - j}$ exhibited antibacterial activity in the range of $15.62-250 \mu \mathrm{g} / \mathrm{mL}$ (Table 2 ). The compounds $3 \mathbf{a}-\mathbf{b}$ were less active than the reference compound Ampicillin against S. aureus, B. subtilis and E. coli. However, compound $\mathbf{3 a}$ was more active than Ampicillin against the $A$. hydrophila and $A$. baumannii strains, and compound $\mathbf{3 b}$ showed the same antibacterial activity as Ampicillin against these two strains. The compounds 4 a-j exhibited lower antibacterial activity than the reference compound Ampicillin against B. subtilis, A. hydrophila and E. coli. Compound $\mathbf{4 b}$ exhibited similar antibacterial activity as Ampicillin against the $S$. aureus strain, with a MIC value of $31.25 \mu \mathrm{g} / \mathrm{mL}$. Furthermore, compounds $\mathbf{4 d}$ and $\mathbf{4 j}$ were more active than Ampicillin against A. baumannii, an opportunistic pathogen with increasing importance in nosocomial infections. The other 2-(thiazol-2-yl)octahydropyrrolo[3,4-c]pyrrole derivatives 4a-c, 4e-i exhibited similar antibacterial activity with Ampicillin against the $A$. baumannii strain.

Table 2. The MIC values $(\mu \mathrm{g} / \mathrm{mL})$ of the tested compounds against the microbial strains.

\begin{tabular}{cccccccccc}
\hline Entry & S. aureus B. subtilis & $\begin{array}{c}\text { A. } \\
\text { hydrophila }\end{array}$ & & E. coli & $\begin{array}{c}\text { A. } \\
\text { baumannii }\end{array}$ & $\begin{array}{c}\text { C. } \\
\text { glabrata }\end{array}$ & $\begin{array}{c}\text { C. } \\
\text { tropicalis }\end{array}$ & $\begin{array}{c}\text { C. } \\
\text { parapsilosis }\end{array}$ & $\begin{array}{c}\text { M. } \\
\text { tuberculosis } \\
\text { H37Rv }\end{array}$ \\
\hline 3a & 125 & 15.62 & $\mathbf{1 5 . 6 2}$ & 250 & $\mathbf{6 2 . 5}$ & 31.25 & 125 & 62.5 & 62.5 \\
3b & 250 & 62.5 & $\mathbf{3 1 . 2 5}$ & 250 & 125 & 31.25 & 125 & 62.5 & 62.5 \\
4a & 62.5 & 125 & 125 & 250 & 125 & 31.25 & 125 & 62.5 & 7.81 \\
4b & $\mathbf{3 1 . 2 5}$ & 125 & 125 & 125 & 125 & 31.25 & 125 & 62.5 & 62.5 \\
\hline
\end{tabular}




\begin{tabular}{cccccccccc}
\hline Entry & S. aureus B. subtilis & $\begin{array}{c}\text { A. } \\
\text { hydrophila }\end{array}$ & E. coli & $\begin{array}{c}\text { A. } \\
\text { baumannii }\end{array}$ & $\begin{array}{c}\text { C. } \\
\text { glabrata }\end{array}$ & $\begin{array}{c}\text { C. } \\
\text { tropicalis }\end{array}$ & $\begin{array}{c}\text { C. } \\
\text { parapsilosis }\end{array}$ & $\begin{array}{c}\text { M. } \\
\text { tuberculosis } \\
H 37 R v\end{array}$ \\
\hline $\mathbf{4 c}$ & 125 & 125 & 125 & 250 & 125 & 31.25 & 125 & 62.5 & 31.25 \\
$\mathbf{4 d}$ & 125 & 125 & 125 & 250 & 62.5 & 31.25 & 62.5 & 62.5 & 31.25 \\
$\mathbf{4 e}$ & 62.5 & 250 & 125 & 250 & 125 & 15.62 & 125 & 31.25 & 31.25 \\
$\mathbf{4 f}$ & 125 & 125 & 125 & 250 & 125 & 62.5 & 250 & 62.5 & 62.5 \\
$\mathbf{4 g}$ & 125 & 250 & 125 & 250 & 125 & 31.25 & 250 & 62.5 & 62.5 \\
$\mathbf{4 h}$ & 125 & 125 & 125 & 250 & 125 & 31.25 & 250 & 62.5 & 62.5 \\
$\mathbf{4 i}$ & 250 & 250 & 125 & 125 & 125 & 31.25 & 125 & 31.25 & 31.25 \\
$\mathbf{4 j}$ & 125 & 125 & 125 & 250 & $\mathbf{6 2 . 5}$ & 31.25 & 125 & 62.5 & 31.25 \\
Ampicillin & $\mathbf{3 1 . 2 5}$ & $\mathbf{0 . 9 8}$ & $\mathbf{3 1 . 2 5}$ & $\mathbf{1 5 . 6 2}$ & $\mathbf{1 2 5}$ & - & - & - & - \\
Fluconazole & - & - & - & - & - & $\mathbf{3 . 9 0}$ & $\mathbf{1 5 . 6 2}$ & $\mathbf{3 . 9 0}$ & - \\
Isoniazid & - & - & - & - & - & - & - & - & $\mathbf{0 . 9 8}$ \\
Ethambutol & - & - & - & - & - & - & - & - & $\mathbf{1 . 9 6}$ \\
\hline
\end{tabular}

MIC: The minimal inhibitory concentrations

The compounds 3a-b, 4a-j showed antifungal activity, in the range of $15.62-250 \mu \mathrm{g} / \mathrm{mL}$, but did not exhibit higher antifungal activity than the reference compound Fluconazole, which exhibited antifungal activity against the fungi with MIC values in the range of $3.90-15.62 \mu \mathrm{g} / \mathrm{mL}$ (Table 2). It can be said that all of the tested compounds exhibited moderate antifungal activity against the mentioned fungi. Compounds 3a-b, 4a-j were also tested against the Mycobacterium tuberculosis H37Rv strain. Compound 4a performed best with a MIC value of $7.81 \mu \mathrm{g} / \mathrm{mL}$, but none of the compounds scored better than the reference drug Isoniazid.

\section{Conclusions}

The synthesis of octahydropyrrolo[3,4-c]pyrrole $\mathrm{N}$-benzoylthiourea derivatives $3 \mathbf{a}-\mathbf{b}$ and the 2-(thiazol-2-yl)octahydropyrrolo[3,4-c]pyrrole derivatives $4 a-j$ was successfully performed in subcritical water as a green solvent. Despite the fact that reactive agents such as isothiocyanates and $\alpha$-haloketones were used, applying subcritical water as a solvent resulted in a significant decrease of reaction times, while maintaining high yields. The synthesized compounds displayed moderate antimicrobial activity against different bacterial and fungal strains and against the Mycobacterium tuberculosis H37Rv strain.

\section{Experimental Section}

General. All chemicals used had high-grade commercial products purchased from Merck or Aldrich and all solvents provided by commercial suppliers had reagent grade quality and were used without further purification. A Varian Scimitar Series 1000 FT-IR spectrophotometer, using horizontal ATR, was used. Nuclear magnetic resonance spectra were determined at $400 \mathrm{MHz}$ on a Bruker Ultrashield Plus Biospin GmbH. Chemical shifts are given in parts per million $(\delta)$ downfield from TMS as internal standard. Flash column chromatography was performed using silica gel 60 (230-400 mesh). Kieselgel columns were packed with silica gel GF254. Melting 
points were determined on a Mettler Toledo MP90 device. Mass spectra were recorded by an Agilent 6460 Triple Quad LC/MS/MS mass spectrometer. High resolution mass spectra were recorded by an LC-MS TOF electrospray ionization technique.

General procedure for the synthesis of octahydropyrrolo[3,4-c]pyrrole derivatives (2a,b). The compounds $2 a, b$ were synthesized using a literature method. ${ }^{42}$ To a stirred solution of methyl 2-(diphenylmethyleneamino) acetate $1(1 \mathrm{mmol})$ in $o$-xylene $(10 \mathrm{~mL})$ was added a solution of $\mathrm{N}$-substituted maleimide $(2 \mathrm{mmol})$ in $o$-xylene $(20 \mathrm{~mL})$ and the reaction mixture was refluxed for $24 \mathrm{~h}$. After completion of the reaction, the mixture was quenched with water and extracted with ethyl acetate. The crude mixture was purified by column chromatography (EtOAc:hexane / 1:5) to give $\mathbf{2} \mathbf{a}, \mathbf{b}$ as colourless crystals.

(1R)-Methyl 5-methyl-4,6-dioxo-3,3-diphenyl-octahydropyrrolo[3,4-c]pyrrole-1-carboxylate (2a). Crystallized from EtOAc:hexane as colorless prisms. Yield, $0.31 \mathrm{~g}, 85 \% . \mathrm{mp} \mathrm{204-206}{ }^{\circ} \mathrm{C}$. IR (cm $\left.{ }^{-1}\right): 3326(\mathrm{~m}), 3053(\mathrm{w}), 2954$ (w), 1743 (s), 1694 (vs). ${ }^{1} \mathrm{H}$ NMR (400 MHz, DMSO-d $): \delta$ 7.51-7.49 (m, 2H, Ar-H), 7.39-7.15 (m, 8H, Ar-H), 4.27 (d, $1 \mathrm{H}, J 7.3 \mathrm{~Hz}, 4-\mathrm{H}), 3.67\left(\mathrm{~s}, 3 \mathrm{H}, \mathrm{OCH}_{3}\right), 3.60$ (dd, $\left.1 \mathrm{H}, J 8.0 \mathrm{~Hz}, 7.4 \mathrm{~Hz}, 3-\mathrm{H}\right), 3.49(\mathrm{~d}, 1 \mathrm{H}, J 8.0 \mathrm{~Hz}, 2-\mathrm{H}), 2.71(\mathrm{~s}$, $\left.3 \mathrm{H}, \mathrm{NCH}_{3}\right) .{ }^{13} \mathrm{CNMR}\left(100 \mathrm{MHz}, \mathrm{DMSO}-d_{6}\right): \delta 176.0$ (C=O), 175.1 (C=O), $170.4(\mathrm{C}=0), 144.9,142.7,128.3(2 \times \mathrm{C})$, $127.3(2 \times \mathrm{C}), 127.2(2 \times \mathrm{C}), 127.0,126.6(2 \times \mathrm{C}), 126.5,71.9,59.0,51.8,51.6,47.9,24.6 . \mathrm{MS}\left(\mathrm{ESI}, \mathrm{M}+\mathrm{H}^{+}\right): \mathrm{m} / z$ $365.2\left(\mathrm{M}+\mathrm{H}^{+}, 100\right)$. HRMS (ESI-TOF-MS): calcd. for $\mathrm{C}_{21} \mathrm{H}_{21} \mathrm{~N}_{2} \mathrm{O}_{4}[\mathrm{MH}]^{+} 365.1501$; found 365.1500 .

(1R)-Methyl 4,6-dioxo-3,3,5-triphenyl-octahydropyrrolo[3,4-c]pyrrole-1-carboxylate (2b). Crystallized from EtOAc:hexane as colorless prisms. Yield, $0.35 \mathrm{~g}, 82 \% . \mathrm{mp} \mathrm{212-214}{ }^{\circ} \mathrm{C} . \mathrm{IR}\left(\mathrm{cm}^{-1}\right): 3273(\mathrm{~m}), 3065(\mathrm{w}), 2961(\mathrm{w})$, 1716 (vs). ${ }^{1} \mathrm{H}$ NMR (400 MHz, $\left.\mathrm{CDCl}_{3}\right): \delta$ 7.47-7.20 (m, 13H, Ar-H), 7.11-7.09 (m, 2H, Ar-H), $4.28(\mathrm{~d}, 1 \mathrm{H}, J 7.5 \mathrm{~Hz}$, 4-H), 3.87 (dd, $1 \mathrm{H}, J 7.2 \mathrm{~Hz}, 6.8 \mathrm{~Hz}, 2-\mathrm{H}), 3.79\left(\mathrm{~s}, 3 \mathrm{H}, \mathrm{OCH}_{3}\right), 3.68$ (dd, 1H, J $\left.7.5 \mathrm{~Hz}, 7.2 \mathrm{~Hz}, 3-\mathrm{H}\right), 2.90(\mathrm{~d}, 1 \mathrm{H}, J 6.8$ $\mathrm{Hz}, \mathrm{NH}) .{ }^{13} \mathrm{CNMR}\left(100 \mathrm{MHz}, \mathrm{CDCl}_{3}\right): \delta 175.0(\mathrm{C}=\mathrm{O}), 174.0(\mathrm{C}=\mathrm{O}), 170.7(\mathrm{C}=\mathrm{O}), 144.5,141.5,131.7,129.0(2 \times \mathrm{C})$, $128.9(2 \times \mathrm{C}), 128.5,128.0(2 \times \mathrm{C}), 127.8,127.7,127.4(2 \times \mathrm{C}), 126.5(2 \times \mathrm{C}), 126.3(2 \times \mathrm{C}), 73.7,60.2,52.9,52.4$, 49.1. $\mathrm{MS}\left(\mathrm{ESI}, \mathrm{M}+\mathrm{H}^{+}\right): \mathrm{m} / z$ 427.2 (M+H', 100). HRMS (ESI-TOF-MS): calcd. for $\mathrm{C}_{26} \mathrm{H}_{23} \mathrm{~N}_{2} \mathrm{O}_{4}[\mathrm{MH}]^{+}$427.1658; found 427.1662.

General procedure for the synthesis of the octahydropyrrolo[3,4-c]pyrrole $\mathbf{N}$-benzoylthiourea derivatives 3a,b in subcritical water. The experiments were carried out in a home-made stainless steel reactor with an internal volume of $200 \mathrm{~mL}$. To synthesize the octahydropyrrolo[3,4-c]pyrrole $N$-benzoylthiourea derivatives 3a-b, the reactor and magnet were firstly flushed with water and acetone, then thoroughly dried with nitrogen gas. The reactor was loaded with octahydropyrrolo[3,4-c]pyrroles 2 a-b $(1 \mathrm{mmol})$, benzoyl isothiocyanate $(2 \mathrm{mmol})$ and $75 \mathrm{~mL}$ of ultra-pure water, after that the reactor cover screws were tightly clamped. After purging the air in the reactor with nitrogen, the internal pressure of the reactor was fixed at 30 bar supplied by nitrogen. Each experiment was performed at $130{ }^{\circ} \mathrm{C}$ for 4 hours under stirring. Finally, the reactor was cooled, depressurized, and the obtained mixture was extracted using dichloromethane (DCM). The crude product was purified by column chromatography (EtOAc:hexane / 1:3) to afford compounds 3a-b.

General procedure for synthesis of the octahydropyrrolo[3,4-c]pyrrole $\mathbf{N}$-benzoylthiourea derivatives (3a,b) in acetone. The octahydropyrrolo[3,4-c]pyrrole $N$-benzoylthiourea derivatives $\mathbf{3 a}, \mathbf{b}$ were also synthesized by modification of a literature method. ${ }^{43,44}$ To a stirred solution of $2 \mathrm{a}(0.37 \mathrm{~g}, 1 \mathrm{mmol})$ or $2 \mathrm{~b}(0.43 \mathrm{~g}, 1 \mathrm{mmol}) \mathrm{in}$ acetone $(20 \mathrm{~mL})$ was added a solution of benzoyl isothiocyanate $(0.33 \mathrm{~g}, 2 \mathrm{mmol})$ in acetone $(10 \mathrm{~mL})$ and the mixture was stirred at reflux temperature for $36 \mathrm{~h}$. After completion of the reaction, the solvent was evaporated under reduced pressure and the crude mixture was purified by column chromatography (EtOAc:hexane / 1:3) to afford compound $\mathbf{3 a} \mathbf{a} \mathbf{b}$. Structures of $\mathbf{3} \mathbf{a}, \mathbf{b}$ were confirmed by NMR and MS techniques.

(1R)-Methyl 2-(benzoylcarbamothioyl)-5-methyl-4,6-dioxo-3,3-diphenyl-octahydropyrrolo[3,4-c]pyrrole-1carboxylate (3a). Crystallized from DCM:hexane as yellow prisms. Yield, $0.45 \mathrm{~g}, 86 \% . \mathrm{mp} 110-112{ }^{\circ} \mathrm{C} . \mathrm{IR}\left(\mathrm{cm}^{-1}\right)$ : 
3351 (m), 3063 (w), 2950 (w), 1741 (m), 1704 (vs). ${ }^{1} \mathrm{H}$ NMR (400 MHz, CDCl $): \delta 8.44$ (s, 1H, N-H), 7.53-7.33 (m, $11 \mathrm{H}, \mathrm{Ar}-\mathrm{H}), 7.12(\mathrm{t}, 2 \mathrm{H}, \mathrm{J} 7.6 \mathrm{~Hz}, \mathrm{Ar}-\mathrm{H}), 6.75(\mathrm{~d}, 2 \mathrm{H}, J 7.6 \mathrm{~Hz}, \mathrm{Ar}-\mathrm{H}), 5.71(\mathrm{~d}, 1 \mathrm{H}, J 10.4 \mathrm{~Hz}, 2-\mathrm{H}), 4.33(\mathrm{~d}, 1 \mathrm{H}, J 9.6$ $\mathrm{Hz}, 4-\mathrm{H}), 3.95\left(\mathrm{~s}, 3 \mathrm{H}, \mathrm{OCH}_{3}\right), 3.86-3.81(\mathrm{~m}, 1 \mathrm{H}, 3-\mathrm{H}), 2.71\left(\mathrm{~s}, 3 \mathrm{H}, \mathrm{NCH}_{3}\right) .{ }^{13} \mathrm{CNMR}\left(100 \mathrm{MHz}, \mathrm{CDCl}_{3}\right): \delta 178.4(\mathrm{C}=\mathrm{S})$, 173.5 (C=O), 172.9 (C=O), 168.5 (C=O), 163.6 (C=O), 140.3, 137.5, 133.0, 132.5, 129.9 ( $2 \times \mathrm{C}), 129.0(2 \times \mathrm{C}), 128.8$ $(2 \times \mathrm{C}), 128.6(2 \times \mathrm{C}), 128.4(2 \times \mathrm{C}), 127.8(2 \times \mathrm{C}), 127.0(2 \times \mathrm{C}), 66.7,63.2,52.8(2 \times \mathrm{C}), 44.1,25.0 . \mathrm{MS}\left(\mathrm{ESI}, \mathrm{M}-\mathrm{H}^{+}\right):$ $\mathrm{m} / \mathrm{z} 526.1\left(\mathrm{M}-\mathrm{H}^{+}, 100\right)$. HRMS (ESI-TOF-MS): calcd. for $\mathrm{C}_{29} \mathrm{H}_{26} \mathrm{~N}_{3} \mathrm{O}_{5} \mathrm{~S}[\mathrm{MH}]^{+}$528.1593; found 528.1597.

(1R)-Methyl

2-(benzoylcarbamothioyl)-4,6-dioxo-3,3,5-triphenyl-octahydropyrrolo[3,4-c]pyrrole-1carboxylate (3b). Crystallized from DCM:hexane as yellow prisms. Yield, $0.47 \mathrm{~g}, 80 \% . \mathrm{mp} 185-187^{\circ} \mathrm{C}$. IR (cm $\left.{ }^{-1}\right)$ : $3356(\mathrm{~m}), 3060$ (w), 2947 (w), 1716 (vs). ${ }^{1} \mathrm{H}$ NMR (400 MHz, CDCl 3 ): $\delta 8.44(\mathrm{~s}, 1 \mathrm{H}, \mathrm{N}-\mathrm{H}), 7.55-7.50(\mathrm{~m}, 6 \mathrm{H}, \mathrm{Ar}-\mathrm{H})$, 7.39-7.32 (m, 8H, Ar-H), 7.15-7.11 (m, 2H, Ar-H), 6.81-6.77 (m, 4H, Ar-H), $5.82(\mathrm{~d}, 1 \mathrm{H}, J 10.3 \mathrm{~Hz}, 2-\mathrm{H}), 4.52(\mathrm{~d}$, $1 \mathrm{H}, J 9.7 \mathrm{~Hz}, 4-\mathrm{H}), 4.08-4.03(\mathrm{~m}, 1 \mathrm{H}, 3-\mathrm{H}), 3.92\left(\mathrm{~s}, 3 \mathrm{H}, \mathrm{OCH}_{3}\right) .{ }^{13} \mathrm{C} \mathrm{NMR}\left(100 \mathrm{MHz}, \mathrm{CDCl}_{3}\right): \delta 178.3(\mathrm{C}=\mathrm{S}), 172.7$ (C=O), 171.8 (C=O), 168.4 (C=O), 163.5 (C=O), 140.6, 137.0, 133.0, 132.5, 131.0, 129.9, 129.3, 129.1 (2x C), 129.0 $(2 \times C), 128.9(2 \times C), 128.7,128.4,128.0,127.8,127.3,127.0,126.4,126.3,126.0$ (3 x C), 79.7, 66.7, 63.1, 52.9,

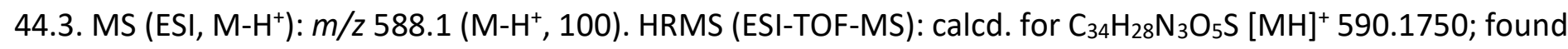
590.1750.

General procedure for the synthesis of the 2-(thiazol-2-yl)-octahydropyrrolo[3,4-c]pyrrole derivatives (4a-j) in subcritical water. The experiments were carried out in steel reactor which was identified in the synthesis of $\mathbf{3} \mathbf{a}, \mathbf{b}$ and the same operations described above were applied. To synthesize the 2-(thiazol-2-yl)octahydropyrrolo[3,4-c]pyrrole derivatives $4 a-j$, the required octahydropyrrolo[3,4-c]pyrrole $N$-benzoylthiourea derivative $3 \mathbf{a}$ or $\mathbf{3 b}(1 \mathrm{mmol})$, an appropriate $\alpha$-haloketone $(1.2 \mathrm{mmol})$ and $75 \mathrm{~mL}$ of ultra-pure water were loaded to the reactor. After purging the air in the reactor with nitrogen, the internal pressure of the reactor was fixed at 30 bar supplied by nitrogen. Each experiment was performed at $130{ }^{\circ} \mathrm{C}$ for 2 hours under stirring. Finally, the reactor was cooled, depressurized, and the obtained mixture was extracted using DCM. The crude product was purified by column chromatography (EtOAc:hexane / 1:4) to afford compounds 4a-j.

General procedure for synthesis of the 2-(thiazol-2-yl)-octahydropyrrolo[3,4-c]pyrrole derivatives (4a-j) in acetone. The 2-(thiazol-2-yl)-octahydropyrrolo[3,4-c]pyrrole derivatives 4a-j were synthesized using a literature method. ${ }^{45}$ To a stirred solution of $3 \mathbf{a}(0.53 \mathrm{~g}, 1 \mathrm{mmol})$ or $3 \mathbf{b}(0.59 \mathrm{~g}, 1 \mathrm{mmol})$ in acetone $(20 \mathrm{~mL})$ was added a solution of an appropriate $\alpha$-haloketone $(1.2 \mathrm{mmol})$ in acetone $(10 \mathrm{~mL})$ and the mixture was stirred at reflux temperature for $18 \mathrm{~h}$. After completion of the reaction, the solvent was evaporated under reduced pressure, quenched with saturated aqueous sodium chloride and extracted with DCM. The crude mixture was purified by column chromatography (EtOAc:hexane/1:4) to afford compound 4a-j. Structures of $4 a-j$ were confirmed by NMR and MS techniques.

(1R)-Methyl 2-(5-benzoyl-4-phenylthiazol-2-yl)-5-methyl-4,6-dioxo-3,3-diphenyl-octahydropyrrolo[3,4c]pyrrole-1-carboxylate (4a). Crystallized from DCM:hexane as yellow prisms. Yield, $0.49 \mathrm{~g}, 78 \% . \mathrm{mp} 145-147^{\circ} \mathrm{C}$ (decomp). IR (cm $\left.{ }^{-1}\right)$ : u 3056 (w), 2924 (w), 1707 (vs). ${ }^{1} \mathrm{H}$ NMR (400 MHz, CDCl $): \delta$ 7.66-7.64(m, 2H, Ar-H), 7.44$7.36(\mathrm{~m}, 10 \mathrm{H}, \mathrm{Ar}-\mathrm{H}), 7.24-7.21(\mathrm{~m}, 3 \mathrm{H}, \mathrm{Ar}-\mathrm{H}), 7.13-7.02(\mathrm{~m}, 5 \mathrm{H}, \mathrm{Ar}-\mathrm{H}), 5.29(\mathrm{~d}, 1 \mathrm{H}, J 10.0 \mathrm{~Hz}, 2-\mathrm{H}), 4.20(\mathrm{~d}, 1 \mathrm{H}, J$ 9.2, 4-H), $3.93\left(\mathrm{~s}, 3 \mathrm{H}, \mathrm{OCH}_{3}\right), 3.84-3.79(\mathrm{~m}, 1 \mathrm{H}, 3-\mathrm{H}), 2.90\left(\mathrm{~s}, 3 \mathrm{H}, \mathrm{NCH}_{3}\right) .{ }^{13} \mathrm{C} \mathrm{NMR}\left(100 \mathrm{MHz}, \mathrm{CDCl}_{3}\right): \delta 189.0(\mathrm{C}=\mathrm{O})$, 174.4 (C=O), 173.4 (C=O), 169.8 (C=O), 166.7, 154.8, 139.7,137.7, 135.0, 134.3, 131.9, $130.3(2 \times C), 129.9(2 \times$ C), $129.5,129.4(2 \times C), 128.8(2 \times C), 128.7(2 \times C), 128.6,128.4(2 \times C), 128.3,127.7(2 \times C), 127.6(2 \times C), 125.6$, 78.4, 65.2, 61.5, 52.6, 45.7, 25.3. MS (ESI, $\left.\mathrm{M}+\mathrm{H}^{+}\right): \mathrm{m} / \mathrm{z} 628.2\left(\mathrm{M}+\mathrm{H}^{+}, 100\right)$. HRMS (ESI-TOF-MS): calcd. for $\mathrm{C}_{37} \mathrm{H}_{30} \mathrm{~N}_{3} \mathrm{O}_{5} \mathrm{~S}[\mathrm{MH}]^{+}$628.1906; found 628.1898.

(1R)-Methyl 2-(5-(4-cyanobenzoyl)-4-phenylthiazol-2-yl)-5-methyl-4,6-dioxo-3,3-diphenyl-octahydropyrrolo [3,4-c]pyrrole-1-carboxylate (4b). Crystallized from DCM:hexane as yellow prisms. Yield, $0.59 \mathrm{~g}, 90 \% . \mathrm{mp} 180-$ $182{ }^{\circ} \mathrm{C}$ (decomp). IR (cm ${ }^{-1}$ ): 3061 (w), 2981 (w), 2231 (w), 1745 (m), 1707 (vs). ${ }^{1} \mathrm{H}$ NMR (400 MHz, CDCl 3 ): $\delta 7.67-$ 
7.65 (m, 2H, Ar-H), 7.44-7.29 (m, 12H, Ar-H), 7.17-7.12 (m, 3H, Ar-H), 7.06-7.03 (m, 2H, Ar-H), 5.30 (d, $1 \mathrm{H}, J 10.0$ $\mathrm{Hz}, 2-\mathrm{H}), 4.21$ (d, $1 \mathrm{H}, \mathrm{J} 9.2 \mathrm{~Hz}, 4-\mathrm{H}), 3.93\left(\mathrm{~s}, 3 \mathrm{H}, \mathrm{OCH}_{3}\right), 3.85-3.81(\mathrm{~m}, 1 \mathrm{H}, 3-\mathrm{H}), 2.90\left(\mathrm{~s}, 3 \mathrm{H}, \mathrm{NCH}_{3}\right) .{ }^{13} \mathrm{C} \mathrm{NMR}(100$ $\mathrm{MHz}_{1} \mathrm{CDCl}_{3}$ ): $\delta 187.1(\mathrm{C}=\mathrm{O}), 174.2(\mathrm{C}=\mathrm{O}), 173.2(\mathrm{C}=\mathrm{O}), 169.7(\mathrm{C}=\mathrm{O}), 167.6,156.3,141.5,139.5,134.8133 .9,131.4$ $(2 \times C), 130.3(2 \times C), 130.0(2 \times C), 129.7,129.6(2 \times C), 129.2,128.9(2 \times C), 128.7(2 \times C), 128.5,128.4(2 \times C)$, $127.7(2 \times \mathrm{C}), 125.4,118.0,114.6,78.5,65.3,61.8,52.7,45.7,25.3 . \mathrm{MS}\left(\mathrm{ESI}, \mathrm{M}+\mathrm{H}^{+}\right): \mathrm{m} / \mathrm{z} 653.2\left(\mathrm{M}+\mathrm{H}^{+}, 100\right)$. HRMS (ESI-TOF-MS): calcd. for $\mathrm{C}_{38} \mathrm{H}_{29} \mathrm{~N}_{4} \mathrm{O}_{5} \mathrm{~S}[\mathrm{MH}]^{+}$653.1859; found 653.1864.

(1R)-Methyl 2-(5-(4-bromobenzoyl)-4-phenylthiazol-2-yl)-5-methyl-4,6-dioxo-3,3-diphenyl-octahydropyrrolo [3,4-c]pyrrole-1-carboxylate (4c). Crystallized from DCM:hexane as yellow prisms. Yield, $0.59 \mathrm{~g}, 83 \% . \mathrm{mp} \mathrm{181-}$

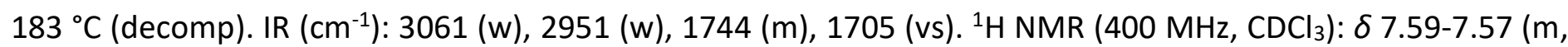
$2 \mathrm{H}, \mathrm{Ar}-\mathrm{H}), 7.36-7.31(\mathrm{~m}, 8 \mathrm{H}, \mathrm{Ar}-\mathrm{H}), 7.19-6.99(\mathrm{~m}, 9 \mathrm{H}, \mathrm{Ar}-\mathrm{H}), 5.22(\mathrm{~d}, 1 \mathrm{H}, J 10.0 \mathrm{~Hz}, 2-\mathrm{H}), 4.14(\mathrm{~d}, 1 \mathrm{H}, J 9.2 \mathrm{~Hz}, 4-$ $\mathrm{H}), 3.86\left(\mathrm{~s}, 3 \mathrm{H}, \mathrm{OCH}_{3}\right), 3.77-3.72(\mathrm{~m}, 1 \mathrm{H}, 3-\mathrm{H}), 2.83\left(\mathrm{~s}, 3 \mathrm{H}, \mathrm{NCH}_{3}\right) .{ }^{13} \mathrm{C} \mathrm{NMR}\left(100 \mathrm{MHz}, \mathrm{CDCl}_{3}\right): \delta 187.7(\mathrm{C}=\mathrm{O})$, 174.3(C=O), 173.3 ( $C=0), 169.7$ ( $C=0), 166.9,155.1,139.6,136.5,134.9,134.1,130.9(4 \times C), 130.3(2 \times C), 129.9$ $(2 \times \mathrm{C}), 129.5,128.8$ (3 x C), 128.7 (2x C), 128.4 (3 x C), 127.7 (2 x C), 126.7, 125.3, 78.4, 65.2, 61.6, 52.6, 45.7, 25.3. MS (ESI, $\left.\mathrm{M}+\mathrm{H}^{+}\right): \mathrm{m} / \mathrm{z} 706.2\left(\mathrm{M}+\mathrm{H}^{+}, 100\right), 708.2\left(\mathrm{M}+\mathrm{H}^{+}, 100\right)$. HRMS (ESI-TOF-MS): calcd. for $\mathrm{C}_{37} \mathrm{H}_{29} \mathrm{BrN}_{3} \mathrm{O}_{5} \mathrm{~S}$ $[\mathrm{MH}]^{+}$706.1011; found 706.0995 .

(1R)-Methyl 2-(5-(2,4-dichlorobenzoyl)-4-phenylthiazol-2-yl)-5-methyl-4,6-dioxo-3,3-diphenyl-octahydropyrrolo[3,4-c]pyrrole-1-carboxylate (4d). Crystallized from DCM:hexane as yellow prisms. Yield, $0.58 \mathrm{~g}, 84 \%$. $\mathrm{mp} 264-266{ }^{\circ} \mathrm{C}$ (decomp). IR (cm $\left.{ }^{-1}\right): 3086(\mathrm{w}), 2918$ (w), $1744(\mathrm{~m}), 1706$ (vs). ${ }^{1} \mathrm{H}$ NMR (400 MHz, CDCl $): \delta 7.65-$ $7.63(\mathrm{~m}, 2 \mathrm{H}, \mathrm{Ar}-\mathrm{H})$, 7.44-7.38 (m, 8H, Ar-H), 7.18-7.14 (m, 3H, Ar-H), 7.07-7.03 (m, 3H, Ar-H), $6.87(\mathrm{~d}, 1 \mathrm{H}, J 8.2$ $\mathrm{Hz}, \mathrm{Ar}-\mathrm{H}), 6.79$ (dd, 1H, J $8.2 \mathrm{~Hz}, 1.9 \mathrm{~Hz}, \mathrm{Ar}-\mathrm{H}), 5.26$ (d, $1 \mathrm{H}, J 10.0 \mathrm{~Hz}, 2-\mathrm{H}), 4.20$ (d, $1 \mathrm{H}, J 9.2 \mathrm{~Hz}, 4-\mathrm{H}), 3.86(\mathrm{~s}, 3 \mathrm{H}$, $\left.\mathrm{OCH}_{3}\right), 3.83-3.78(\mathrm{~m}, 1 \mathrm{H}, 3-\mathrm{H}), 2.88\left(\mathrm{~s}, 3 \mathrm{H}, \mathrm{NCH}_{3}\right) .{ }^{13} \mathrm{C} \mathrm{NMR}\left(100 \mathrm{MHz}, \mathrm{CDCl}_{3}\right): \delta 185.5(\mathrm{C}=\mathrm{O}), 174.2(\mathrm{C}=\mathrm{O}), 173.3$ $(C=O), 169.5(C=O), 168.1,157.4,139.5,136.7,136.2,134.7,133.8,132.7,130.6,130.3(2 \times C), 129.6,129.5(3 \times$ C), $128.8(3 \times \mathrm{C}), 128.7(2 \times \mathrm{C}), 128.4,129.3(2 \times \mathrm{C}), 127.4(3 \times \mathrm{C}), 126.2,78.6,65.2,61.7,52.6,45.6,25.3$. MS (ESI, $\left.\mathrm{M}+\mathrm{H}^{+}\right): \mathrm{m} / \mathrm{z} 696.2\left(\mathrm{M}+\mathrm{H}^{+}, 100\right), 697.2\left(\mathrm{M}+\mathrm{H}^{+}, 45\right), 698.2\left(\mathrm{M}+\mathrm{H}^{+}, 75\right)$. HRMS (ESI-TOF-MS): calcd. for $\mathrm{C}_{37} \mathrm{H}_{28} \mathrm{Cl}_{2} \mathrm{~N}_{3} \mathrm{O}_{5} \mathrm{~S}[\mathrm{MH}]^{+}$696.1127; found 696.1120.

(1R)-Methyl 5-methyl-4,6-dioxo-2-(5-(2-oxo-2H-chromene-3-carbonyl)-4-phenylthiazol-2-yl)-3,3-diphenyloctahydropyrrolo[3,4-c]pyrrole-1-carboxylate (4e). Crystallized from DCM:hexane as yellow prisms. Yield, 0.63 g, 91\%. mp 200-202 ${ }^{\circ} \mathrm{C}$ (decomp). IR (cm $\left.{ }^{-1}\right): 3063$ (w), 2981 (w), 1733 (m), 1708 (vs). ${ }^{1} \mathrm{H} \mathrm{NMR} \mathrm{(400} \mathrm{MHz,} \mathrm{CDCl} 3$ ): $\delta 7.67(\mathrm{~m} \mathrm{2H}, \mathrm{Ar}-\mathrm{H}), 7.44-7.41(\mathrm{~m}, 9 \mathrm{H}, \mathrm{Ar}-\mathrm{H}), 7.30-7.26(\mathrm{~m}, 3 \mathrm{H}, \mathrm{Ar}-\mathrm{H}), 7.23-7.21(\mathrm{~m}, 1 \mathrm{H}, \mathrm{Ar}-\mathrm{H}), 7.18-7.11(\mathrm{~m}, 2 \mathrm{H}$, Ar-H), 7.07-6.99 (m, 3H, Ar-H), $5.28(\mathrm{~d}, 1 \mathrm{H}, J 10.0 \mathrm{~Hz}, 2-\mathrm{H}), 4.23(\mathrm{~d}, 1 \mathrm{H}, J 9.2 \mathrm{~Hz}, 4-\mathrm{H}), 3.87\left(\mathrm{~s}, 3 \mathrm{H}, \mathrm{OCH}_{3}\right), 3.84-$ $3.79(\mathrm{~m}, 1 \mathrm{H}, 3-\mathrm{H}), 2.87\left(\mathrm{~s}, 3 \mathrm{H}, \mathrm{NCH}_{3}\right) .{ }^{13} \mathrm{C} \mathrm{NMR}\left(100 \mathrm{MHz}, \mathrm{CDCl}_{3}\right): \delta 182.6(\mathrm{C}=0), 174.2(\mathrm{C}=0), 173.2(\mathrm{C}=0), 169.6$ $(C=0), 168.1(C=0), 157.7(2 \times C), 154.0,142.8,139.4,134.6,134.5,132.9,130.3(2 \times C), 129.6(3 \times C), 128.9(3 \times$ C), $128.7(2 \times C), 128.5(2 \times C), 128.4(2 \times C), 127.8(2 \times C), 127.2,127.1,124.4,117.8,116.4,78.6,65.3,61.8$, 52.6, 45.7, 25.3. MS (ESI, $\left.\mathrm{M}+\mathrm{H}^{+}\right): \mathrm{m} / z 696.2\left(\mathrm{M}+\mathrm{H}^{+}, 100\right)$. HRMS (ESI-TOF-MS): calcd. for $\mathrm{C}_{40} \mathrm{H}_{30} \mathrm{~N}_{3} \mathrm{O}_{7} \mathrm{~S}[\mathrm{MH}]^{+}$ 696.1804; found 696.1797.

(1R)-Methyl 2-(5-benzoyl-4-phenylthiazol-2-yl)-4,6-dioxo-3,3,5-triphenyl-octahydropyrrolo[3,4-c]pyrrole-1carboxylate (4f). Crystallized from DCM:hexane as yellow prisms. Yield, $0.44 \mathrm{~g}, 74 \% \mathrm{mp} 251-253{ }^{\circ} \mathrm{C}$ (decomp). IR (cm ${ }^{-1}$ ): 3063 (w), $2981(w), 1750(\mathrm{~m}), 1717$ (vs). ${ }^{1} \mathrm{H}$ NMR (400 MHz, CDCl $): \delta 7.78$ (brs, 2H, Ar-H), 7.49-7.35 (m, 16H, Ar-H), 7.25-7.22 (m, 3H, Ar-H), 7.13-7.02 (m, 4H, Ar-H), $5.39(\mathrm{~d}, 1 \mathrm{H}, J 9.9 \mathrm{~Hz}, 2-\mathrm{H}), 4.38(\mathrm{~d}, 1 \mathrm{H}, J 9.4,4-$ $\mathrm{H}), 3.99-3.94(\mathrm{~m}, 1 \mathrm{H}, 3-\mathrm{H}), 3.93\left(\mathrm{~s}, 3 \mathrm{H}, \mathrm{OCH}_{3}\right) .{ }^{13} \mathrm{C} \mathrm{NMR}\left(100 \mathrm{MHz}_{\mathrm{CDCl}}\right): \delta 189.0(\mathrm{C}=0), 173.5(\mathrm{C}=0), 172.3$ (C=O), 169.8 (C=O), 166.5, 154.8, 139.8, 137.7, 134.7, 134.3, 131.9, 131.4, $130.6(2 \times C), 129.9(2 \times C), 129.5$, $129.4(2 \times \mathrm{C}), 129.2(2 \times \mathrm{C}), 128.9,128.8(2 \times \mathrm{C}), 128.7,128.6(2 \times \mathrm{C}), 128.5,128.0,127.7(2 \times \mathrm{C}), 127.6(2 \times \mathrm{C})$, 127.3, 126.4, $126.3(2 \times \mathrm{C}), 78.7,65.5,61.6,52.7,45.7 . \mathrm{MS}\left(\mathrm{ESI}, \mathrm{M}+\mathrm{H}^{+}\right): \mathrm{m} / z$ 690.2 $\left(\mathrm{M}+\mathrm{H}^{+}, 100\right)$. HRMS (ESI-TOFMS): calcd. for $\mathrm{C}_{42} \mathrm{H}_{32} \mathrm{~N}_{3} \mathrm{O}_{5} \mathrm{~S}[\mathrm{MH}]^{+} 690.2063$; found 690.2053 . 
(1R)-Methyl 2-(5-(4-cyanobenzoyl)-4-phenylthiazol-2-yl)-4,6-dioxo-3,3,5-triphenyl-octahydropyrrolo[3,4c]pyrrole-1-carboxylate (4g). Crystallized from DCM:hexane as yellow prisms. Yield, $0.63 \mathrm{~g}, 88 \% . \mathrm{mp} 166-168{ }^{\circ} \mathrm{C}$ (decomp). IR (cm ${ }^{-1}$ ): 3061 (w), 2923 (w), 2229 (w), 1751 (m), 1719 (vs). ${ }^{1} \mathrm{H}^{\mathrm{N} M R}\left(400 \mathrm{MHz}, \mathrm{CDCl}_{3}\right): \delta 7.80$ (brs, $2 \mathrm{H}, \mathrm{Ar}-\mathrm{H}), 7.48-7.38(\mathrm{~m}, 13 \mathrm{H}, \mathrm{Ar}-\mathrm{H}), 7.31-7.26(\mathrm{~m}, 2 \mathrm{H}, \mathrm{Ar}-\mathrm{H}), 7.17-7.13(\mathrm{~m}, 3 \mathrm{H}, \mathrm{Ar}-\mathrm{H}), 7.07-7.03(\mathrm{~m}, 4 \mathrm{H}, \mathrm{Ar}-\mathrm{H})$, $5.42(\mathrm{~d}, 1 \mathrm{H}, J 10.0 \mathrm{~Hz}, 2-\mathrm{H}), 4.42(\mathrm{~d}, 1 \mathrm{H}, J 9.4 \mathrm{~Hz}, 4-\mathrm{H}), 4.04-4.00(\mathrm{~m}, 1 \mathrm{H}, 3-\mathrm{H}), 3.93\left(\mathrm{~s}, 3 \mathrm{H}, \mathrm{OCH}_{3}\right) .{ }^{13} \mathrm{C} \mathrm{NMR}(100$ $\mathrm{MHz}_{\mathrm{CDCl}}$ ): $\delta 187.1$ (C=O), 173.3 (C=O), 172.1 (C=O), 169.7 (C=O), 167.5, 156.4, 141.5, 139.6, 134.8 133.9, 131.4 $(2 \times C), 131.3,130.6(2 \times C), 130.0(2 \times C), 129.7,129.6(2 \times C), 129.2(3 \times C), 129.0,128.9(2 \times C), 128.8(2 \times C)$, 128.6, $128.5(2 \times \mathrm{C}), 127.8(2 \times \mathrm{C}), 126.2(2 \times \mathrm{C}), 125.5,118.0,114.6,78.9,65.6,61.8,52.8,45.7 . \mathrm{MS}\left(\mathrm{ESI}, \mathrm{M}+\mathrm{H}^{+}\right)$: $\mathrm{m} / \mathrm{z} 715.2\left(\mathrm{M}+\mathrm{H}^{+}, 100\right)$. HRMS (ESI-TOF-MS): calcd. for $\mathrm{C}_{43} \mathrm{H}_{31} \mathrm{~N}_{4} \mathrm{O}_{5} \mathrm{~S}[\mathrm{MH}]^{+} 715.2015$; found 715.2029.

(1R)-Methyl 2-(5-(4-bromobenzoyl)-4-phenylthiazol-2-yl)-4,6-dioxo-3,3,5-triphenyl-octahydropyrrolo[3,4c]pyrrole-1-carboxylate (4h). Crystallized from DCM:hexane as yellow prisms. Yield, $0.58 \mathrm{~g}, 75 \% . \mathrm{mp} 165-167^{\circ} \mathrm{C}$ (decomp). IR (cm ${ }^{-1}$ ): 3059 (w), 2956 (w), 1745 (m), 1714 (vs). ${ }^{1} \mathrm{H}$ NMR (400 MHz, CDCl 3 ): $\delta 7.79$ (brs, 2H, Ar-H), 7.50-7.37 (m, 12H, Ar-H), 7.22-7.16 (m, 6H, Ar-H), 7.11-7.05 (m, 4H, Ar-H), $5.42(\mathrm{~d}, 1 \mathrm{H}, J 10.0 \mathrm{~Hz}, 2-\mathrm{H}), 4.41(\mathrm{~d}$, $1 \mathrm{H}, J 9.4 \mathrm{~Hz}, 4-\mathrm{H}), 4.03-3.99(\mathrm{~m}, 1 \mathrm{H}, 3-\mathrm{H}), 3.93\left(\mathrm{~s}, 3 \mathrm{H}, \mathrm{OCH}_{3}\right) .{ }^{13} \mathrm{C} \mathrm{NMR}\left(100 \mathrm{MHz}, \mathrm{CDCl}_{3}\right): \delta 187.2(\mathrm{C}=\mathrm{O}), 173.4$ (C=O), 172.2 (C=O), 169.8 (C=O), 166.8, 155.2, 139.7, 136.5, 134.6, 134.1, 131.4, $130.9(3 \times C), 130.6(2 \times C)$, $129.9(2 \times \mathrm{C}), 129.6,129.2(3 \times \mathrm{C}), 128.9,128.8$ ( $4 \times \mathrm{C}), 128.5(3 \times \mathrm{C}), 127.7(2 \times \mathrm{C}), 126.7,126.2(3 \times \mathrm{C}), 125.4$, 78.8, 65.6, 61.7, 52.7, 45.7. MS (ESI, $\left.\mathrm{M}+\mathrm{H}^{+}\right): \mathrm{m} / \mathrm{z} 768.2\left(\mathrm{M}+\mathrm{H}^{+}, 100\right), 770.2\left(\mathrm{M}+\mathrm{H}^{+}, 100\right)$. HRMS (ESI-TOF-MS): calcd. for $\mathrm{C}_{42} \mathrm{H}_{31} \mathrm{BrN}_{3} \mathrm{O}_{5} \mathrm{~S}[\mathrm{MH}]^{+} 768.1168$; found 768.1150.

(1R)-Methyl 2-(5-(2,4-dichlorobenzoyl)-4-phenylthiazol-2-yl)-4,6-dioxo-3,3,5-triphenyl-octahydropyrrolo[3,4-c]pyrrole-1-carboxylate (4i). Crystallized from DCM:hexane as yellow prisms. Yield, $0.61 \mathrm{~g}, 81 \%$. $\mathrm{mp} \mathrm{160-}$ $162{ }^{\circ} \mathrm{C}$. IR (cm ${ }^{-1}$ ): 3063 (w), 2952 (w), 1750 (m), 1717 (vs). ${ }^{1} \mathrm{H} \mathrm{NMR}\left(400 \mathrm{MHz}, \mathrm{CDCl}_{3}\right.$ ): $\delta 7.77$ (brs, 2H, Ar-H), 7.45$7.36(\mathrm{~m}, 12 \mathrm{H}, \mathrm{Ar}-\mathrm{H}), 7.16-7.14(\mathrm{~m}, 2 \mathrm{H}, \mathrm{Ar}-\mathrm{H}), 7.07-7.01(\mathrm{~m}, 5 \mathrm{H}, \mathrm{Ar}-\mathrm{H}), 6.88(\mathrm{~d}, 1 \mathrm{H}, J 8.2 \mathrm{~Hz}, \mathrm{Ar}-\mathrm{H}), 6.79(\mathrm{dd}, 1 \mathrm{H}, J$ $8.2 \mathrm{~Hz}, 1.9 \mathrm{~Hz}, \mathrm{Ar}-\mathrm{H}), 5.36(\mathrm{~d}, 1 \mathrm{H}, J 10.0 \mathrm{~Hz}, 2-\mathrm{H}), 4.37$ (d, 1H, J $9.4 \mathrm{~Hz}, 4-\mathrm{H}), 3.97-3.92(\mathrm{~m}, 1 \mathrm{H}, 3-\mathrm{H}), 3.86(\mathrm{~s}, 3 \mathrm{H}$, $\left.\mathrm{OCH}_{3}\right) .{ }^{13} \mathrm{C} \mathrm{NMR}\left(100 \mathrm{MHz}, \mathrm{CDCl}_{3}\right): \delta 185.5(\mathrm{C}=\mathrm{O}), 173.4(\mathrm{C}=\mathrm{O}), 172.2(\mathrm{C}=\mathrm{O}), 169.6(\mathrm{C}=0), 168.0,157.5,139.6$, 136.7, 136.2, 134.3, 133.8, 132.7, 131.3, 130.6, 130.5, 129.7, 129.5 (3 x C), 129.2 (2 x C), 129.0, $128.9(2 \times C)$, $128.8(2 \times \mathrm{C}), 128.6,128.5(2 \times \mathrm{C}), 128.0,127.4(2 \times \mathrm{C}), 127.3,126.4,126.3,126.2(2 \times \mathrm{C}), 79.0,65.6,61.8,52.7$, 45.7. $\mathrm{MS}\left(\mathrm{ESI}, \mathrm{M}+\mathrm{H}^{+}\right): \mathrm{m} / \mathrm{z} 758.2\left(\mathrm{M}+\mathrm{H}^{+}, 100\right), 759.2\left(\mathrm{M}+\mathrm{H}^{+}, 45\right), 760.2\left(\mathrm{M}+\mathrm{H}^{+}, 75\right)$. HRMS (ESI-TOF-MS): calcd. for $\mathrm{C}_{42} \mathrm{H}_{30} \mathrm{Cl}_{2} \mathrm{~N}_{3} \mathrm{O}_{5} \mathrm{~S}[\mathrm{M} \mathrm{H}]^{+} 758.1283$; found 758.1280 .

(1R)-Methyl 4,6-dioxo-2-(5-(2-oxo-2H-chromene-3-carbonyl)-4-phenylthiazol-2-yl)-3,3,5-triphenyl-octahydropyrrolo[3,4-c]pyrrole-1-carboxylate (4j). Crystallized from DCM:hexane as yellow prisms. Yield, $0.68 \mathrm{~g}$, 90\%. mp 310-312 ${ }^{\circ} \mathrm{C}$ (decomp). IR (cm $\left.{ }^{-1}\right): 3063(\mathrm{w}), 2981$ (w), 1749 (m), 1719 (vs). $\left.{ }^{1} \mathrm{H} \mathrm{NMR} \mathrm{(400} \mathrm{MHz,} \mathrm{CDCl} 3\right): \delta$ 7.81 (brs, 2H, Ar-H), 7.49-7.36 (m, 13H, Ar-H), 7.31-7.26 (m, 2H, Ar-H), 7.21-7.12 (m, 3H, Ar-H), 7.08-6.99 (m, 5H, $\operatorname{Ar}-\mathrm{H}), 5.40(\mathrm{~d}, 1 \mathrm{H}, J 10.0 \mathrm{~Hz}, 2-\mathrm{H}), 4.45(\mathrm{~d}, 1 \mathrm{H}, J 9.4 \mathrm{~Hz}, 4-\mathrm{H}), 4.04-3.99(\mathrm{~m}, 1 \mathrm{H}, 3-\mathrm{H}), 3.88\left(\mathrm{~s}, 3 \mathrm{H}, \mathrm{OCH}_{3}\right) .{ }^{13} \mathrm{C} \mathrm{NMR}$ $\left(100 \mathrm{MHz}_{\mathrm{C}} \mathrm{CDCl}_{3}\right): \delta 182.6(\mathrm{C}=\mathrm{O}), 173.3(\mathrm{C}=\mathrm{O}), 172.1(\mathrm{C}=\mathrm{O}), 169.6(\mathrm{C}=\mathrm{O}), 167.9$ (C=O), 157.7, 157.6, 154.0, 142.8, 139.5, 134.5, 134.3, 132.9, 131.3, $130.6(2 \times C), 129.6(3 \times C), 129.2(3 \times C), 129.0,128.9(2 \times C), 128.8,128.6$, $128.5(3 \times \mathrm{C}), 127.8(2 \times \mathrm{C}), 127.2(2 \times \mathrm{C}), 126.2(3 \times \mathrm{C}), 124.4,117.8,116.5,79.0,65.6,61.8,52.7,45.7 . \mathrm{MS}$ (ESI, $\left.\mathrm{M}+\mathrm{H}^{+}\right): \mathrm{m} / \mathrm{z} 758.2\left(\mathrm{M}+\mathrm{H}^{+}, 100\right)$. HRMS (ESI-TOF-MS): calcd. for $\mathrm{C}_{45} \mathrm{H}_{32} \mathrm{~N}_{3} \mathrm{O}_{7} \mathrm{~S}[\mathrm{MH}]^{+} 758.1961$; found 758.1951.

Antibacterial activity. The antibacterial properties of the compounds were determined in duplicate against Bacillus subtilis (ATCC 6633) and Staphylococcus aureus (ATCC 25925) as Gram-positive bacterial strains and Acinetobacter baumannii (ATCC 02026), Aeromonas hydrophila (ATCC 95080) and Escherichia coli (ATCC 25923) as Gram-negative bacterial strains, acquired from the Refik Saydam Hıfzıssıhha Institute, Ankara, Turkey. The compounds 4a-j were dissolved in DMSO to prepare the stock solutions, and then diluted in Triptic soy broth and Mueller-Hinton broth to obtain an initial concentration of $1000 \mu \mathrm{g} / \mathrm{mL}$. Further dilutions of $4 a-j$ and the control drug ampicillin were prepared at concentrations of 500-0.12 $\mu \mathrm{g} / \mathrm{mL}$. A negative control for the effect of 
DMSO on microbial growth was performed, using inoculated broth supplemented with DMSO at the same dilutions used for the test compounds, and was determined to be inactive.

Antifungal activity. The antifungal properties of $4 a-j$ were determined against three yeast strains (C. parapsilosis ATCC 22019, C. glabrata ATCC 90030 and C. tropicalis ATCC 750) using the microdilution broth procedure 46 according to the NCCLS standard document M27-A2. ${ }^{47}$ The fungal strains were acquired from Refik Saydam Hifzıssıhha Institute, Ankara, Turkey. Fluconazole (Sigma, F8929) was used as positive control. Antifungal activity was determined in RPMI 1640 Medium (Sigma, R6504) which was buffered to pH 7.0 with 0.165 M 3-(Nmorpholino)-propanesulfonic acid (MOPS, Sigma, M1254) as described in the NCCLS document. A working suspension of the fungal strains was made by a 1:100 dilution followed by a 1:20 dilution of the stock suspension with RPMI 1640 medium. Stock solutions of $4 a-j$ and reference antifungal agent in DMSO were prepared at a concentration of $1000 \mu \mathrm{g} / \mathrm{mL}$ and filtered through a $0.22 \mu \mathrm{m}$ membrane filter. Serial two fold dilutions of these solutions and reference antifungal agent were prepared in a 96-well microtitre plate using $100 \mu$ I RPMI 1640 medium. The tested concentration range was $500-0.12 \mu \mathrm{g} / \mathrm{mL}$. A growth control containing no antibiotic and a sterility control without inoculum were included in each plate. The working inoculum suspension (100 $\mu$ l) was added to each plate. The plates were incubated at $35{ }^{\circ} \mathrm{C}$ for $48 \mathrm{~h}$ in ambient air. The MIC is determined as the lowest concentration of a compound that visually inhibits growth of the organism.

Antimycobacterial activity. Antimycobacterial properties of $4 a-j$ were determined using the resazurin microtitre assay (REMA) plate method. ${ }^{48}$

Culture medium: 7H9-S medium was used for the REMA plate method, consisting of Middlebrook 7H9 broth (BBL, Becton Dickinson and Company, Sparks, MD, USA) containing $0.1 \%$ casitone, $0.5 \%$ glycerol and $10 \%$ oleic acid-albumin-dextrose-catalase (OADC, BBL, Becton Dickinson and Company, Sparks, MD, USA).

Resazurin reagent: Resazurin sodium salt powder (Sigma R7017) was dissolved in distilled water at a concentration of $0.01 \%(\mathrm{w} / \mathrm{v})$ and sterilized by filtration through a $0.22 \mu \mathrm{m}$ membrane filter (Ministar, Sartorius Stedim Biotech $\mathrm{GmbH}$, Goettingen, Germany) to obtain a working solution which was stored at $4{ }^{\circ} \mathrm{C}$ for up to 1 week.

The REMA plate method was used in duplicate as described by Nateche et al. ${ }^{48}$ with minor changes. Ethambutol (EMB) (Sigma, E4630) and isoniazid (INH) (Sigma, 13377) were used as control agents. M. tuberculosis H37Rv was used as the standard strain and was acquired from the Refik Saydam National Public Health Agency, National Tuberculosis Reference Laboratory, Ankara, Turkey. Compounds $\mathbf{4 a - j}$ and the reference compounds were dissolved in DMSO at a concentration of $1000 \mu \mathrm{g} / \mathrm{mL}$ and filtered through a $0.22 \mu \mathrm{m}$ membrane filter to obtain stock solutions. Serial twofold dilutions of these solutions were prepared in a 96-well microtitre plate using 100 $\mu \mathrm{L}$ 7H9-S. The tested concentration range was 500-0.12 $\mu \mathrm{g} / \mathrm{mL}$. A growth control containing no antibiotic and a sterility control without inoculum were included in each plate. The H37Rv inoculum was prepared by resuspending a loopful of the Lowenstein-Jensen culture medium in a tube containing $5 \mathrm{ml}$ 7H9-S medium with several glass beads. After vortexing the tube for 2 minutes, sediment was allowed to form for 30 minutes. The supernatant was transferred to a second sterile tube and the turbidity was adjusted to match a McFarland standard No. 1. This suspension was further diluted 1:20 in 7H9-S. The plates were inoculated with $100 \mu \mathrm{L}$ suspension, sealed in plastic bags and incubated at $37{ }^{\circ} \mathrm{C}$ in a normal atmosphere. After 7 days of incubation, 30 $\mu \mathrm{L}$ resazurin working solution was added to each well and the plates were incubated for 24 hours at $37^{\circ} \mathrm{C}$. The results were determined visually. Bacterial growth gives reduction of resazurin and is indicated by a change in color from blue to pink. In order to conclude a positive result, the color change which indicates growth has to be comparable to that observed in the positive growth control. The MIC was defined as the lowest concentration of solution that prevented a full color change of resazurin from blue to pink. 


\section{Acknowledgements}

This work was funded by Mersin University Research Fund (Project No: BAP 2016-2-AP3-1800).

\section{References}

1. Arshadi, S.; Vessally, E.; Edjlali, L.; Hosseinzadeh-Khanmiri, R.; Ghorbani-Kalhor, E. Beilstein J. Org. Chem. 2017, 13, 625-638.

https://doi.org/10.3762/bjoc.13.61

2. Das, D.; Sikdar, P.; Bairagi, M. Eur. J. Med. Chem. 2016, 109, 89-98.

https://doi.org/10.1016/i.ejmech.2015.12.022

3. Arora, P.; Narang, R.; Nayak, S.K.; Singh, S.K.; Judge, V. Med. Chem. Res. 2016, 25, 1717-1743. https://doi.org/10.1007/s00044-016-1610-2

4. Wang, M.; Zhang, J.; He, S.; Yan, X. Mar. Drugs 2017, 15, Article Number: 126, 1-19.

5. Abhale, Y.K.; Shinde, A.; Deshmukh, K.K.; Nawale, L.; Sarkar, D.; Mhaske, P.C. Med. Chem. Res. 2017, 26, 2557-2567.

https://doi.org/10.1007/s00044-017-1955-1

6. Vaickelionienè, R. ; Mickevičius, V. ; Vaickelionis, G. ; Stasevych, M. ; Komarovska-Porokhnyavets, O. ; Novikov, V. Arkivoc 2015, (v), 303-318.

http://dx.doi.org/10.3998/ark.5550190.p009.170

7. Kaur, K.; Kumar, V.; Beniwal, V.; Kumar, V.; Kumar, N.; Sharma, V.; Jaglan, S. Med. Chem. Res. 2016, 25, 2237-2249.

https://doi.org/10.1007/s00044-016-1663-2

8. Argyropoulou, I.; Geronikaki, A.; Vicini, P.; Zani, F. Arkivoc 2009, (vi), 89-102.

http://dx.doi.org/10.3998/ark.5550190.0010.611

9. Bhalerao, M.B.; Dhumal, S.T.; Deshmukh, A.R.; Nawale, L.U.; Khedkar, V; Sarkar, D.; Mane, R.A. Bioorg Med Chem Lett. 2017, 27, 288-294.

https://doi.org/10.1016/j.bmcl.2016.11.056

10. Dhumal, S.T.; Deshmukh, A.R.; Bhosle, M.R.; Khedkar, V.M.; Nawale, L.U.; Sarkar, D.; Mane, R.A. Bioorg. Med. Chem. Lett. 2016, 26, 3646-3651.

https://doi.org/10.1016/j.bmcl.2016.05.093

11. Surineni, G.; Yogeeswari, P.; Sriram, D.; Kantevari, S. Bioorg. Med. Chem. Lett. 2016, 26, 3684-3689. https://doi.org/10.1016/j.bmcl.2016.05.085

12. Titus, S.; Sreejalekshmi, K.G. Med. Chem. Res. 2018, 27, 23-36.

https://doi.org/10.1007/s00044-017-2039-y

13. Olawode, E.O.; Tandlich, R.; Prinsloo, E.; Isaacs, M.; Hoppe, H.; Seldon, R.; Warner, D.F.; Steenkamp, V.; Kaye, P.T. Arkivoc 2016, (vi), 284-296.

https://doi.org/10.24820/ark.5550190.p009.905

14. Chen, B-C.; Zhao, R.; Wang, B.; Droghini, R.; Lajeunesse, J.; Sirard, P.; Endo, M.; Balasubramanian, B.; Barrish, J.C. Arkivoc 2010, (vi), 32-38.

http://dx.doi.org/10.3998/ark.5550190.0011.604 
15. Madni, M.; Hameed, S.; Ahmed, M.N.; Tahir, M.N.; Al-Masoudi, N.A.; Pannecouque, C. Med. Chem. Res. 2017, 26, 2653-2665. https://doi.org/10.1007/s00044-017-1963-1

16. Al-Masoudi, N.A.; Al-Haidery, N.; Faili, N.T.; Pannecouque, C. Arkivoc 2010,(ix), 185-195. http://dx.doi.org/10.3998/ark.5550190.0011.918

17. Sahin, O.; Ozdemir, U.O.; Seferoglu, N.; Genc, Z.K.; Kaya, K.; Aydiner, B.; Tekin, S.; Seferoglu, Z. J. Photoch. Photobio. B. 2018, 178, 428-439.

18. Sun, C.; Su, R.; Bie, J.; Sun, H.; Qiao, S.; Ma, X.; Sun, R.; Zhang, T. Dyes Pigments 2018, 149, 867-875. https://doi.org/10.1016/j.dyepig.2017.11.031

19. Saraswat, P.; Jeyabalan, G.; Hassan, M.Z.; Rahman, M.U.; Nyola, N.K. Synth. Commun. 2016, 46, 16431664. https://doi.org/10.1080/00397911.2016.1211704

20. Shi, S.; Zhu, S.; Gerritz, S.W.; Esposito, K.; Padmanabha, R.; Li, W.; Herbst, J.J.; Wong, H.; Shu, Y.Z.; Lam, K.S.; Sofia, M.J. Bioorg. Med. Chem. Lett. 2005, 15, 4151-4154.

https://doi.org/10.1016/j.bmcl.2005.06.004

21. Milen, M.; Abranyi-Balogh, P.; Keglevich, G. Curr. Org. Synth. 2014, 11, 889-901. https://doi.org/10.2174/1570179411666140818210247

22. Nural, Y.; Gemili, M.; Seferoglu, N.; Sahin, E.; Ulger, M.; Sari, H. J. Mol. Struct. 2018, 1160, 375-382. https://doi.org/10.1016/j.molstruc.2018.01.099

23. Dandia, A.; Khan, S.; Soni, P.; Indora, A.; Mahawar, D.K.; Pandya, P.; Chauhan, C.S. Bioorg. Med. Chem. Lett. 2017, 27, 2873-2880. https://doi.org/10.1016/i.bmcl.2017.04.083

24. Ersen, D.; Ulger, M.; Mangelinckx, S.; Gemili, M.; Sahin, E.; Nural, Y. Med. Chem. Res. 2017, 26, 2152-2160. https://doi.org/10.1007/s00044-017-1907-9

25. Kang, I-J.; Hsu, S-J.; Yang, H-Y.; Yeh, T-K.; Lee, C-C.; Lee, Y-C.; Tian, Y-W.; Song, J-S.; Hsu, T-A.; Chao, Y-S.; Yueh, A.; Chern, J-H. J. Med. Chem. 2017, 60, 228-247.

https://doi.org/10.1021/acs.jmedchem.6b00962

26. Liang, J.; Labadie, S.; Zhang, B.; Ortwine, D.F.; Patel, S.; Vinogradova, M.; Kiefer, J.R.; Mauer, T.; Gehling, V.S.; Harmange, J-C.; Cummings, R.; Lai, T.; Liao, J.; Zheng, X.; Liu, Y.; Gustafson, A.; Van der Porten, E.; Mao, W.; Liederer, B.M.; Deshmukh, G.; An, L.; Ran, Y.; Classon, M.; Trojer, P.; Dragovich, P.S.; Murray, L. Bioorg. Med. Chem. Lett., 2017, 27, 2974-2981.

https://doi.org/10.1016/j.bmcl.2017.05.016

27. Elías-Rodríguez, P.; Moreno-Clavijo, E.; Carrion-Jimenez, S.; Carmona, A.T.; Moreno-Vargas, A.J.; Caffa, I.; Montecucco, F.; Cea, M.; Nencioni, A.; Robina, I. Arkivoc 2014,(iii), 197-214.

http://dx.doi.org/10.3998/ark.5550190.p008.492

28. Ghannay, S.; Bakari, S.; Ghabi, A.; Kadri, A.; Msaddek, M.; Aouadi, K. Bioorg. Med. Chem. Lett. 2017, 27, 2302-2307.

https://doi.org/10.1016/j.bmcl.2017.04.044

29. Kamiński, K.; Rzepka, S.; Obniska, J. Bioorg. Med. Chem. Lett. 2011, 21, 5800-5803. https://doi.org/10.1016/j.bmcl.2011.07.118

30. Bharkavi, C.; Kumar, S.V.; Ali, M.A.; Osman, H.; Muthusubramanian, S.; Perumal, S. Bioorg. Med. Chem. Lett. 2017, 27, 3071-3075.

https://doi.org/10.1016/j.bmcl.2017.05.050 
31. Gemili, M.; Sari, H.; Ulger, M.; Sahin, E.; Nural, Y. Inorg. Chim. Acta 2017, 463, 88-96. https://doi.org/10.1016/j.ica.2017.04.026

32. Thaqi, A.; Scott, J.L.; Gilbert, J.; Sakoff, J.A.; McCluskey, A. Eur. J. Med. Chem. 2010, 45, 1717-1723. https://doi.org/10.1016/j.ejmech.2010.01.004

33. Sirin, O.Z.; Demirkol, O.; Akbaslar, D.; Giray, E.S. J. Supercrit. Fluid. 2013, 81, 217-220. https://doi.org/10.1016/i.supflu.2013.05.014

34. Bubalo, M.C.; Vidović, S.; Redovniković, I.R.; Jokić, S. J. Chem. Technol. Biotechnol. 2015, 90, 1631-1639. https://doi.org/10.1002/jctb.4668

35. Yabalak, E.; Adiguzel, S.K.; Adiguzel, A.O.; Ergene, R.S.; Tuncer, M.; Gizir, A.M. Desalin. Water. Treat. 2017, 81, 186-198.

https://doi.org/10.5004/dwt.2017.21089

36. Yabalak, E.; Gizir, A.M. J. Serb. Chem. Soc. 2013, 78, 1013-1022. https://doi.org/10.2298/JSC120321123Y

37. Yabalak, E.; Görmez, Ö.; Gözmen, B.; Gizir, A.M. Int. J. Ind. Chem. 2015, 6, 23-29. https://doi.org/10.1007/s40090-014-0028-2

38. Kus, N.S. Tetrahedron 2012, 68, 949-958. https://doi.org/10.1016/j.tet.2011.10.070

39. Ramos, L.; Kristenson, E.M.; Brinkman, U.A.T. J. Chromatogr. A 2002, 975, 3-29. https://doi.org/10.1016/S0021-9673(02)01336-5

40. Pirmoradi, M.; Kastner, J.R. ACS Sustain. Chem. Eng. 2017, 5, 1517-1527. https://doi.org/10.1021/acssuschemeng.6b02201

41. Kim, M.; Lee, B.Y.; Ham, H.C.; Han, J.; Nam, S.W.; Lee, H-S, Park, J.H.; Choi, S.; Shin, Y. J. Supercrit. Fluid. 2016, 111, 8-13. https://doi.org/10.1016/i.supflu.2016.01.011

42. Khlebnikov, A.F.; Novikov, M.S.; Khlebnikov, V.A.; Kostikov, R.R. Russ. J. Org. Chem. 2001, 37, 507-512. https://doi.org/10.1023/A:1012425701412

43. Gumus, I.; Solmaz, U.; Binzet, G.; Keskin, E.; Arslan, B.; Arslan, H. J. Mol. Struct. 2018, 1157, 78-88. https://doi.org/10.1016/j.molstruc.2017.12.017

44. Binzet, G.; Gumus, I.; Dogen, A.; Florke, U.; Kulcu, N.; Arslan, H. J. Mol. Struct. 2018, 1161, 519-529. https://doi.org/10.1016/j.molstruc.2018.02.073

45. Thomas, K.K.; Reshmy, R.; Ushadevi, K.S. J. Indian Chem. Soc. 2007, 84, 1016-1019

46. Sonmez, M.; Celebi, M.; Berber, I. Eur. J. Med. Chem. 2010, 45, 1935-1940. https://doi.org/10.1016/i.ejmech.2010.01.035

47. NCCLS. Reference Method for Broth Dilution Antifungal Susceptibility Testing of Yeasts; Approved Standard-Second Edition. NCCLS document M27-A2 (ISBN 1-56238-469-4). NCCLS, 940 West Valley Road, Suite 1400, Wayne, Pennsylvania 19087-1898 USA, 2002.

48. Nateche, F.; Martin, A.; Baraka, S.; Palomino, J.C.; Khaled, S.; Portaels, F. J. Med. Microbiol. 2006, 55, 857860.

https://doi.org/10.1099/jmm.0.46513-0 